\title{
3D shape reconstruction from relief calculation: application to human body
}

\author{
I. Leandry ${ }^{1, a}$, C. Breque $^{2}$, P. Lacouture ${ }^{2}$ and V. Valle ${ }^{1}$ \\ ${ }^{1}$ Axe PEM/ PPRIME, UPR 3346, CNRS, Université de Poitiers, ENSMA, BP 30179 \\ 86962 Futuroscope Chasseneuil Cedex, France. \\ ${ }^{2}$ Axe RoBioSS/ PPRIME, UPR 3346, CNRS, Université de Poitiers, ENSMA, BP 30179 \\ 86962 Futuroscope Chasseneuil Cedex, France.
}

\begin{abstract}
In experimental mechanics especially in experimental biomechanics, evaluating a volume object is a major research axe. In mechanics, $3 D$ reconstruction is made from different optical methods like stereovision [1] or structured light methods. In biomechanics, it is achieved from tomography techniques or from models which divide human body into 15, 16 and 17 polyarticulated solids [2][3]. Those solids are simple volumes (cylinders, spheres). In this review, a method of 3D reconstruction from two calculated relief is proposed. Then, the geometric parameters are extracted using an algorithm and compared to the real dimension of various objects. The first test consist in studying the feasibility of the $3 D$ reconstruction using a real upper arm and a mannequin. In a second test an analysis of a sphere and two cylinders which have different dimensions is performed. The aim of those last tests is to evaluate the performances of the proposed method, to show distortion volume problems and to propose solutions.
\end{abstract}

\section{INTRODUCTION}

In our society, ergonomy, safety and health are essential. For example, in the domain of vehicle conceptions, 3D- virtual mannequin are used to improve the comfort and safety. The most popular 3D-mannequin is employed in crash test simulation. In this case, human body modelling are needed. This is also the case of the orthosis fabrication for the health domain. Since the last century, many approaches were proposed to build pertinent human body models. The mains consist, for the first one, in reducing human body to his gravity center. This approach is simple but no information on the movement can be given. The purpose of the second model is to calculate the articular strength and moment - driving element are situated in the joints. The last one does not consider that the driving elements are situated in the joints but driving elements are muscle.

These methods of modelling are based on three principle assumptions[4] :

- The hypotheses implied by the geometric simplifications, by stiff segments, by perfect connections (joints offering no resistances), are not relevant of the real human body.

- The position of the articular center is not accurately known. [5]

- Errors involve with the anthropometrical [6] and geometric data of the segment [7].

For the case of health domain, anthropometrical data precision is very essential for the model because it influences the diagnostic and so the correlation between the treatment and the diseases. Moreover, a good data accuracy allows building prosthesis and orthosis, adapted to different morphotypes.

To compute a completed neuro-musculoskeletal model, it is necessary to have good input data. So, another recent approach has been proposed by Hanavan [3]: human body is, in this case, divided in 15 segments modelled by simple geometric solids (cone, cylinder, and sphere). The weight distribution among the model segment is determined by regression equations based on experimental result. From this model and knowing the segment mass, gravity center and inertial moment can be

\footnotetext{
${ }^{\text {a }}$ E-Mail : ismaelle.leandry@ univ-poitiers.fr
}

This is an Open Access article distributed under the terms of the Creative Commons Attribution-Noncommercial License 3.0, which permits unrestricted use, distribution, and reproduction in any noncommercial medium, provided the original work is properly cited. 
calculated. Another model based on the previous one and proposed by Hatze is composed by 17 body segments. The obesity, the sexes and the pregnancy of a patient are taken into account [2]. It is a realist model but 242 parameters are needed for all measurements (estimated measurement time : 1h20). Those last two models are 3D but irregular forms of real body segments are modelled by simple geometric volumes. To compare them from each other, those models need accurate data, a simple and fast measurement method. Optical measurement techniques are also adapted to this application.

The proposed study concerns the calculation of the human body segment volumes by an optical method. In this work, a measurement method is chosen, a measurement procedure is proposed and the accuracy is evaluated.

\section{EXPERIMENTAL PROCEDURES}

\subsection{Principle}

3D measurement techniques are non-contact and are based on optical principle. The most employed are:

- The time-of-flight optical ranging system: [8-9]

- The laser scanner: [10]

- The interferometry: [11-12]

- The stereoscopy : [13]

- The moiré : [14-15]

- The structured light projection: [16-17-18]

For the presented study, the structured light projection is chosen for its simplicity of use. Moreover, it can be adapted to fast motion measurements and therefore, dynamics problems can be considered for future developments. This method allows calculating the relief of large-dimension objects and has a accuracy which can vary from 0.01 to some millimetres (function of the dimension of the analysed object). Its principle consists in projecting a pattern of fringes and in recording an image of the object with another point of view [19]. To obtain better measurement accuracies, a phase shifting method is used [15], and for an absolute determination of relief, a code-gray method is employed [16]. This technique is usually used in experimental mechanics for the measurement of reliefs and out-of-plane displacements of structures [17-19].In most of cases, the employed setup is made up of one camera and one fringe projector (for example a video-projector). To reconstruct a volume, a minimum of two systems is needed. Each system can measure the relief of one face of the body. For our case, the experimental setup is made up of 2 projection moiré systems (two cameras and two video-projectors). To minimize the number of uncalculated points (shadow zones), video-projectors are placed over the CCD camera. The characteristic parameters of the setup are:

- Camera-object distance : $4 \mathrm{~m}$

- Camera elevation : $0.825 \mathrm{~m}$

- Elevation of video-projectors: $1.74 \mathrm{~m}$

- Resolution of cameras: $1280 \times 1024$ pixel $^{2}$

- Resolution of video-projectors: $1024 \times 768$ pixel $^{2}$ 


\subsection{Calibration procedure}

Calibration is made with a reference plan for each measurement system. Figure 1 and 2 present the process of calibration [19]. At first, the acquisition of projected fringes on a reference plan is done. Then, another acquisition of same plan, rotated with an angle $\beta$, is made. For each step, 16 images are acquired ( 8 for the phase shifting technique and 8 for the gray-code calculation). The work space is so defined by the volume generated by the rotation of the calibration plane (figure 2). Calibration is only valid in this workspace.

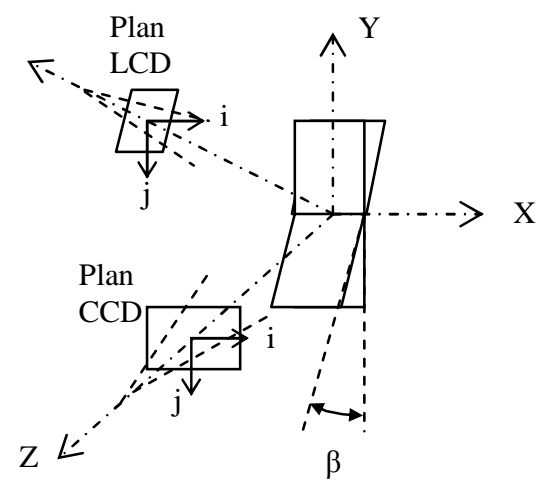

Fig 1- calibration of measurement system

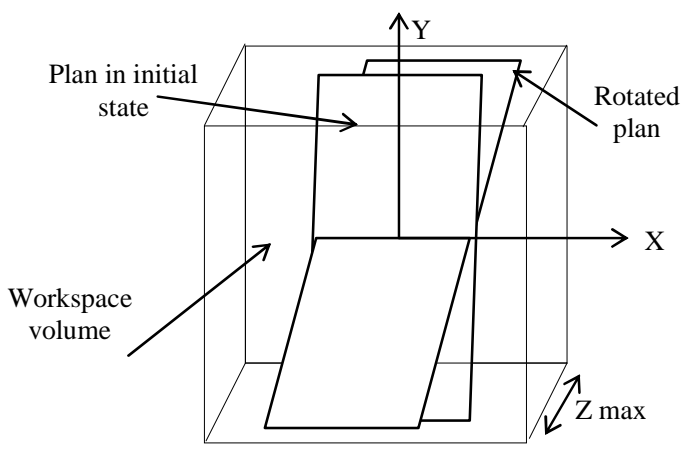

Fig 2- definition of the workspace volume

To combine the two measured reliefs, five small spheres are placed in the visualized workspace. Those spheres are fixed and their positions are supposed perfectly known as it is shown in figure 3 .

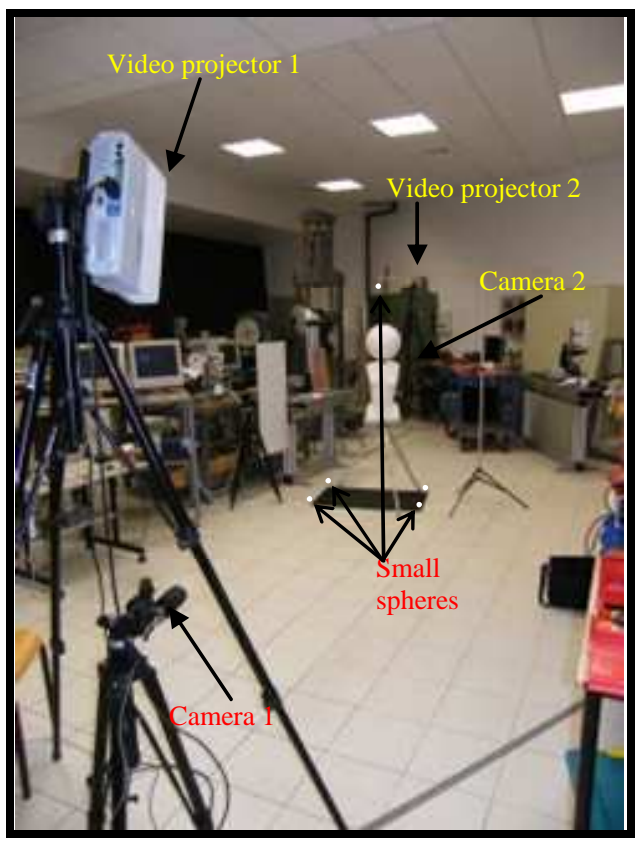

Fig 3- Photography of the complete setup with the 5 spheres situation 
The spheres coordinates are used to define the transformation allowing the coordinate transfer from coordinate system 2 to coordinate system 1 . This change of coordinate system needs the determination of a translation and a rotation movement. Figure 4 exposes the diagram of transformation.

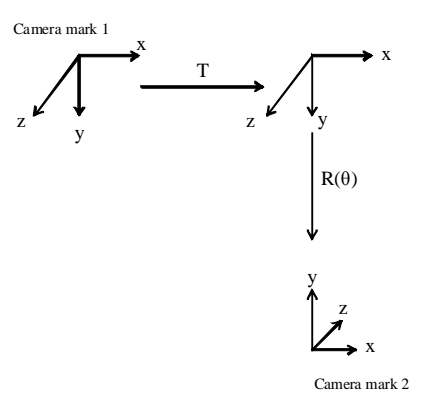

Fig 4- diagram of transformation

The transformation matrix $M$ can be written by:

$$
X_{1}=M \cdot X_{2}
$$

with

$$
\begin{aligned}
M & =T \cdot R(\theta) \\
M & =\left(\begin{array}{ll}
R & T \\
0 & 1
\end{array}\right): \text { tranformation } \\
T & =\left\{T_{x}, T_{y}, T_{z}\right\}^{t}: \text { translation } \\
R & =\left(\begin{array}{lll}
a_{11} & a_{12} & a_{13} \\
a_{21} & a_{22} & a_{23} \\
a_{31} & a_{32} & a_{33}
\end{array}\right): \text { rotation }
\end{aligned}
$$

From the sphere coordinates, $M$ components (12 components) are calculated by using a classical algorithm based on the Gauss method. When this procedure is made, the transformation can be applied to all relief acquired in the coordinate system 2 and can give the relief in the coordinate system 1 .

\section{RESULTS}

The aim of the first presented tests is to validate the setup and the analysis process, to evaluate the feasibility of the technique, the number of necessary acquisition systems and the compatibility of the method with human body. For that, and after the calibration procedure, a white mannequin composed by a trunk and a spherical head is placed in the acquisition volume. On figure 5 , the volume of the mannequin is shown. One can note that the volume is well calculated. 

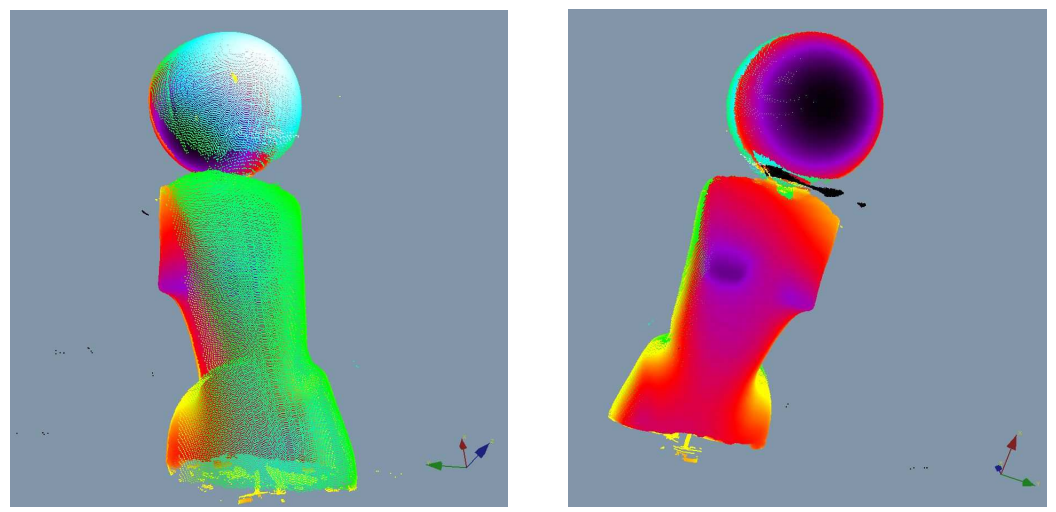

Fig 5- 3D reconstruction of the studied trunk and head

A second test is presented to evaluate the performance of the technique in the case of real human segment. Difference with de previous test is on the colour of the analysed object. On the figure 6 , the volume of a real arm is also well reconstructed. The skin natural colour of the body segment does not generate noise on the measured signal.

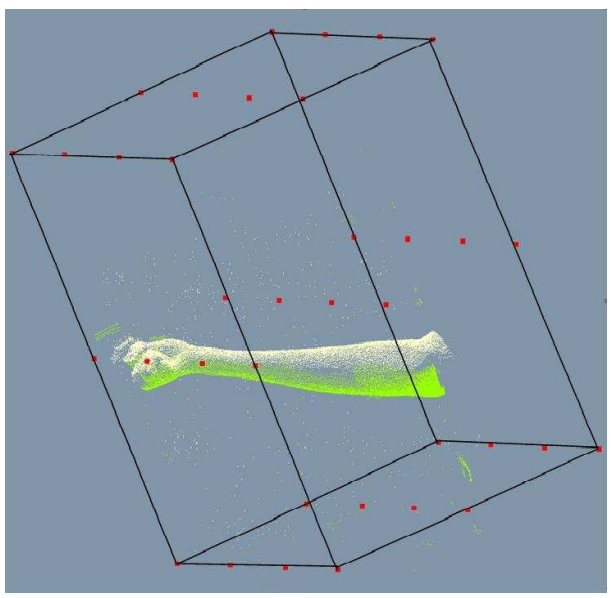

Fig 6- 3D reconstruction of an upper arm- Red points allow the workspace volume visualisation

In figure 5 and 6 , various colours are used to make difference between the two calculated reliefs. The result of these initial tests is promising. After these tests, it will be interesting to evaluate the accuracy of the method. For that, three simple volumes are tested (one sphere and two cylinders) (figure 7). In these cases, the relief is calculated by the same procedure than the previous one. 


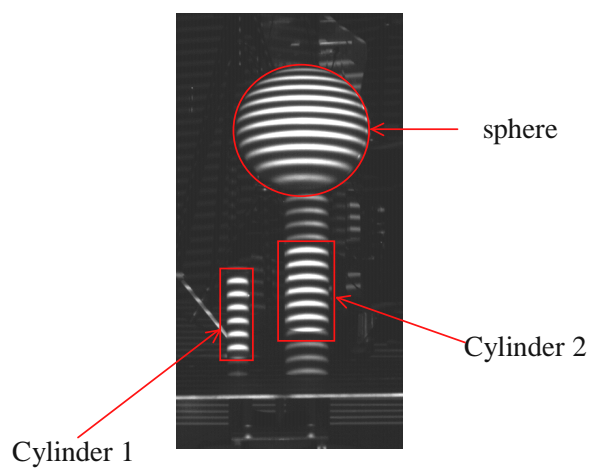

Fig 7- photography of tested objects

From these reliefs, the first table presents the accuracy results along the $\mathrm{z}$ axis. For that, the radius of curvature is determined for each tested object ( $\operatorname{tab} 1)$.

\begin{tabular}{|c|c|c|c|}
\hline solids & measured radius & calculated radius & difference \\
\hline cylinder 1 & 25 & 32.9 & 7.9 \\
\hline cylinder 2 & 50 & 53.22 & 3.22 \\
\hline sphere(XoZ) & 150 & 143.52 & 6.48 \\
\hline sphere(YoZ) & 150 & 142.2 & 7.8 \\
\hline
\end{tabular}

Tab 1 evaluation of the radius; radius is in $\mathrm{m}$

The difference between the calculated and the measured radius can reached the 8 millimeters. The second table shows the accuracy along the y axis. For that, the dimension calculated is the length of the two cylinders.

\begin{tabular}{|c|c|c|c|}
\hline solids & calculated lenght & measured lenght & difference \\
\hline cylinder 1 & 205.79 & 210 & 4.21 \\
\hline cylinder 2 & 206.58 & 210 & 3.42 \\
\hline
\end{tabular}

Tab 2 evaluation of the length; length is in $\mathrm{m}$

These tests show that measurement accuracies along the y axis is two time better than those obtained along the $\mathrm{z}$ axis. As the aim of this work is to retrieve the volume of body segment, the volume error can be calculated on table 3 from the radius and length measurements.

\begin{tabular}{|c|c|c|c|}
\hline solids & real volume & calculated volume & difference \\
\hline cylinder 1 & 0.41 & 0.71 & 0.30 \\
\hline cylinder 2 & 1.65 & 1.87 & 0.22 \\
\hline sphere & 14.13 & 12.21 & -1.92 \\
\hline
\end{tabular}

Tab 3- evaluation of volume error; volume is in $\mathrm{m}^{3}$ 
These tables show that obtained accuracy values are far from those found in literature. This proves the presence of volume distortion problems. These distortions are the direct consequence of the calibration process. Indeed, in these works, Brèque et al [19] have developed calibration equations only valuable if the camera-object distance is 100 times greater than the object dimension. But, as one can see previously in this paper, this condition is not respected. Results are so perturbed by the use of calibration equations in an inappropriate case. It can be supposed that the equation degree are not enough to describe accurately the proposed configuration. In the used calibration, equation degree is 1 along the $\mathrm{x}$ axis, 2 along the $\mathrm{y}$ axis and 1 along the $\mathrm{z}$ axis. To improve results it will be interesting to increase the equation degree or to change the calibration procedure.

\section{CONCLUSION}

In this review, a procedure of 3D reconstruction has been exposed. The structured light projection method was chosen for three principle reasons : its simplicity, the calculation of a large dimension object, and the possible extension to the motion measurement. Indeed, this technique presents a simplicity of implementation and the speed of recording allows the clinical application, in particular on old patient and on handicapped patient.

The first test show that 3D reconstruction does not need a lot of means: a minimum of two measurement systems composed by two cameras and two video-projectors. It can be applied to human body: natural skins generate no additional noise. The second test has proved the presence of a volume distortion. However, the accuracy has been evaluated around the $8 \mathrm{~mm}$. To conclude, this method is appropriated for an application in biomechanics but another calibration procedure must be developed to give an appropriate measurement accuracy.

\section{REFERENCES}

1. E. Grosso, G. Sandini, M. Tistarelli, IEEE SMC,19,nº, 6 , $1465-1476$

2. H. Hatze, Journal of Biomechanics ,13, 833-843 (1980)

3. Hanavan, Technical report ,64-102 (2008)

4. M. G Ishac, D. A. Winter, ISB 93 Paris, 1, 618-619 (1993)

5. T. Monnet, E. Dessally, M. Begon, C. Vallée, P. Lacouture, Journal of Biomechanics, 40, $3487-$ 3492 (2007)

6. M. Begon, P. Lacouture, Science et Motricité, 54,12-23 (2005)

7. M. Begon, P. Lacouture, Science et Motricité, 55, 36-60 (2005)

8. J. S. Massa, Applied optics, 37, n³1, 7298-7304 (1998)

9. I. Moring, H. Ailisto, Optics and Laser in Engineering, 10, 149-160 (1989)

10. C. P. Keferstein, M. Marxer, Sensor Review, 18,3, 183-187 (1998)

11. H.M. Shang, Y. Hung, W.D. Luo, F.Chen, Optical Engineering, 39 (2000)

12. L. Humbert, F. Brémand, V. Valle, M. Cottron, International Journal of Solids and Structures, 37, 5493-5504 (2000) 


\section{EPJ Web of Conferences}

13. E.R. Valstar ,F.W. de Jong, H.A. Vrooman, P.M. Rozing, J.H.C. Reiber, Journal of Biomechanics, 34, 715-722 (2001)

14. L. Pirodda, Optics and Laser in Engineering, 21 (1982)

15. F. Brémand, Optics and Lasers in Engineering, 21, 49-60 (1994)

16. L. Guisser, R. Payrissat, S. Castan, Image and Vision computing, 463-491 (2000)

17. F. Bremand, P. Doumalin, J.C. Dupre, F. Hesser, V. Valle, 13th International Conference on Experimental Mechanics (2007)

18. Y. Morimoto, M. Fujisaa, 33, 3709-3714 (1994)

19. C. Brèque ,J.C. Dupré, F. Brémand, Optics and Laser in Engineering, 241-260 (2002) 